López-Bedoya, J.; Vernetta-Santana, M.; Lizaur Girón, P.: Martínez-Patiño, M.J. y Ariza-Vargas, L. (2019). Comparación de técnicas de entrenamiento de flexibilidad (FNP) con y sin electroestimulación / Comparison of Flexibility Training Techniques (Pnf) with and without Electrostimulation. Revista Internacional de Medicina y Ciencias de la Actividad Física y el $\begin{array}{lllll}\text { Deporte } & \text { vol. } & 19 & \text { (74) } & \text { pp. }\end{array}$ Http://cdeporte.rediris.es/revista/revista74/artcomparacion1011.htm

DOI: http://doi.org/10.15366/rimcafd2019.74.007

\title{
ORIGINAL
}

\section{COMPARACIÓN DE TÉCNICAS DE ENTRENAMIENTO DE FLEXIBILIDAD (FNP) CON Y SIN ELECTROESTIMULACIÓN}

\section{COMPARISON OF FLEXIBILITY TRAINING TECHNIQUES (PNF) WITH AND WITHOUT ELECTROSTIMULATION}

\author{
López-Bedoya, J.1; Vernetta-Santana, M..'; Lizaur Girón, P.1: Martínez- \\ Patiño, M.J. ${ }^{2}$ y Ariza-Vargas, L. ${ }^{3}$ \\ 1 Departamento de Educación Física y Deportes, Facultad de Ciencias del Deporte, Universidad \\ de of Granada, Granada (España). ilopezb@ugr.es, vernetta@ugr.es, plizaur@ugr.es \\ 2 Departamento de Didácticas Especiales, Facultad de Educación Física y Ciencias del Deporte, \\ Universidad de Vigo (España) mipatino@uvigo.es \\ 3 Departamento de Educación Física y Artística, Facultad de Ciencias de la Educación, \\ Universidad de Córdoba (España) eo1arval@uco.es
}

Código UNESCO / UNESCO Code: 241106 Fisiología del ejercicio / Exercise Physiology

Clasificación Consejo de Europa / Council of Europe classification: 17. Otras (Entrenamiento deportivo) / Other (Sports training).

Recibido 9 de mayo de 2016 Received May 9, 2016

Aceptado 6 de junio de 2017 Accepted June 6, 2017

\section{RESUMEN}

El objetivo de este estudio fue analizar el efecto de la técnica de estiramiento Contract-Relax Agonist Contract con (CRAC+EE) y sin (CRAC) electroestimulación sobre la mejora y retención del rango de movimiento activo (AROM) y pasivo (PROM) de cadera en flexión, en extremidad inferior dominante. 34 estudiantes universitarios fueron asignados a tres grupos: control, CRAC+EE y CRAC. AROM y PROM fueron evaluados antes, una vez finalizada $y$ tras dos semanas de la finalización del entrenamiento. El entrenamiento tuvo una duración de cuatro semanas, a razón de tres sesiones semanales. El 
ANOVA mostró un aumento muy significativo de AROM $(p<0,001$ y $p<0,005)$ y PROM $(p<0,001$ y $p<0,01)$ en ambos grupos experimentales respectivamente. En la retención, se mantienen valores superiores con respecto a la medida pretest. Como conclusión, la aplicación de CRAC+EE y CRAC mejoró AROM y PROM, siendo además efectivas en la retención de sendos rangos de movimiento.

PALABRAS CLAVE: Flexibilidad, Entrenamiento, Facilitación Neuromuscular Propioceptiva, Electroestimulación.

\begin{abstract}
The objective of this study was to analyze the effect of the Contract-Relax Agonist Contract stretching technique with $(\mathrm{CRAC}+\mathrm{EE})$ and without (CRAC) electrostimulation on the improvement and retention of active movement range (AROM) and passive (PROM) of hip in flexion, in dominant lower extremity. 34 university students were assigned to three groups: control, CRAC+EE and CRAC. AROM and PROM were evaluated before, once completed and after 2 weeks of completion the training. The training lasted 4 weeks, with 3 sessions per week. The ANOVA showed a very significant increase in AROM $(p<0.001$ and $p<0.005)$ and PROM $(p<0.001$ and $p<0.01)$ in both experimental groups respectively. In the retention, higher values are maintained with respect to the pre-test measurement. In conclusion, the application of CRAC++EE and CRAC improved AROM and PROM, being also effective in the retention of two types of range of motion.
\end{abstract}

KEY WORDS: Training, Flexibility, Proprioceptive Neuromuscular Facilitation, Electrostimulation

\title{
INTRODUCCIÓN
}

La flexibilidad como capacidad física es una cualidad determinante que aumenta y optimiza el aprendizaje y el rendimiento en el movimiento deportivo (Alter, 2004). Es la cualidad que permite al ser humano movilizar los segmentos corporales alcanzando grandes rangos de movimiento (ROM). El entrenamiento de la flexibilidad (solicitud máxima) puede realizarse mediante tres técnicas básicas de estiramientos: estáticas, dinámicas y estiramientos con precontracción (Page, 2012). Este último grupo, implica una contracción previa al músculo a estirar, siendo la técnica más común la Facilitación Neuromuscular Propioceptiva (PNF), la cual ha mostrado su eficacia en la mejora del Rango de movimiento tanto activo como pasivo en diferentes articulaciones dentro de la comunidad deportiva (Sundquist, 1996; McAtee y Charland, 2000; Kenric, 2003; López-Bedoya, Vernetta, Robles y Ariza, 2013; García- Manso, López-Bedoya, Rodríguez-Matoso, Ariza-Vargas, Rodríguez-Ruiz y Vernetta, 2015).

Se trata de un método que está destinado a promover o acelerar la respuesta del mecanismo neuromuscular por medio de la estimulación de los 
propioceptores (Voss, lonta y Meyers, 2004). Se aprovecha de reflejos naturales (reflejo de inhibición autógena) para conseguir inhibir la contracción muscular y así alcanzar mayores ROM (McAtee y Charland, 2000). La eficacia de la FNP se sustenta sobre la utilización de unos patrones de movimiento en masa denominados espirales-diagonales y sobre unas técnicas específicas que secuencian la actuación muscular durante la realización de los patrones de movimiento.

Para Barnet (2006), la electroestimulación implica la transmisión de impulsos eléctricos a través de electrodos de superficie para estimular las neuronas motoras en la periferia provocando contracciones musculares.

Son muchos los autores que han empleado la EE en el deporte con el fin de lograr objetivos diversos: mejorar la fuerza y resistencia muscular; aumentar la masa muscular; agilizar la recuperación después de un esfuerzo; prevenir y mejorar la rehabilitación de lesiones (Maffiuletti, Dugnani, Folz, Di Pierno y Mauro, 2002; Martín, Millet, Lattier y Perrod, 2004; Pombo, Rodríguez, Barnada, Brunet y Requena, 2004; Naffiuletti, Zory, Miotti, Pellegrino, Jubeau y Bottinelli, 2006). Sin embargo, son pocos los estudios existentes donde la estimulación eléctrica permite generar una contracción análoga a la fisiológica haciendo que el impulso eléctrico inducido dé lugar a la contracción muscular para ver su efecto sobre el ROM (Acosta, López-Bedoya y Vernetta, 1998; Pérez y Álamo, 2001; López-Bedoya, Goméz-Landero, Jiménez y Vernetta, 2002; De Hoyo y Sañudo 2006; Espejo, Maya, Cardero y Albornoz, 2012).

Entre las numerosas técnicas de PNF destinadas a la mejora del ROM, las más usadas en la literatura científica relacionada con el entrenamiento deportivo son las denominadas Contract-Relax (CR) y Hold-Relax (HR) (Surburg y Schrader, 1997; Acosta et al., 1998; Adler, Berkers y Buck, 2002; Voss et al., 2004; López Bedoya et al., 2013), siendo menos estudiada la técnica denominada Contract-Relax-Agonist Contract (CRAC) (Etnyre y Abraham, 1986; McAtee y Charland, 2000). Esta última técnica se ejecuta de modo muy parecido a la CR, es decir, en su primera parte, se realiza una contracción isotónica concéntrica del músculo que hay que estirar, seguido por una fase de relajación y posteriormente una contracción isométrica del músculo opositor, seguido de un estiramiento activo hacia el nuevo rango de movimiento (McAtee y Charland, 2000; Norris, 2007; Ayala, Sainz de Baranda y Cejudo, 2012). Se cree que esta contracción activa del músculo opositor al estiramiento estimula la inhibición reciproca del músculo diana, permitiendo con ello un estiramiento más profundo.

Son varios los estudios que han comprobado su eficacia sobre el incremento del rango de movimiento activo y pasivo a largo plazo (Etnyre y Abraham, 1986; Sundquist 1996; McAtee y Charland, 2000). Sin embargo, existe poca documentación de su efecto cuando se induce la contracción agonista mediante electroestimulación (Acosta et al., 1988; Pérez y Álamo, 2001; López Bedoya et al., 2002). 


\section{OBJETIVOS}

El objetivo fundamental del estudio fue evaluar el efecto a largo plazo del entrenamiento de la flexibilidad mediante la técnica CRAC sin y con inducción de la última fase de contracción mediante electroestimulación, sobre el rango de movimiento pasivo y activo de la musculatura isquiotibial en jóvenes deportistas. Igualmente, se pretende conocer cuál de los dos tipos entrenamientos permite mantener la mejora obtenida, tras un periodo de inactividad de dos semanas.

\section{MATERIAL Y MÉTODO}

\section{Sujetos}

Un total de 34 jóvenes deportistas, estudiantes universitarios en Ciencias del Deporte, especialidad Gimnasia Artística, con un rango de edad comprendido entre 20 y 23 años (edad $=21,80 \pm 1,20$ años; masa corporal $=66,81 \pm 9,60 \mathrm{~kg}$; talla $=170,52 \pm 8,22 \mathrm{~cm}$ ), fueron asignados a tres grupos: control, Contract-RelaxContracción de Agonista inducida a través de electroestimulación (CRAC+EE) y CRAC, mediante técnicas de bloqueo en función de los datos obtenidos en el pre-test. Todos los participantes fueron plenamente informados de los procedimientos y posibles riesgos antes de obtener su consentimiento por escrito. Ninguno de ellos padecía lesiones en la musculatura indicada ni ninguna otra dolencia.

El estudio se realizó de acuerdo con la Declaración de Helsinki y fue aprobado por el Comité de Ética de la Universidad de Granada.

\section{Diseño experimental}

Se utilizó un diseño factorial de medidas repetidas, con tres niveles en el factor inter-grupo (control, CRAC+EE, CRAC) y tres niveles en el factor intragrupo (medida pre-, post- y re-test). Las variables dependientes fueron el rango de movimiento pasivo (PROM) y el rango de movimiento activo (AROM) de la flexión de cadera de pierna dominante.

\section{Instrumentación y equipo}

Se utilizó una cámara fotográfica digital estándar (Nikon, Coolpix S500, Nikon Corporation, Chiyoda-ku, Tokio, Japón, http://www.nikon.com/) para capturar la imagen durante las pruebas de evaluación ASLRT y PSLRT y posteriormente recoger los datos de los ángulos. Por otro lado, en la fase de entrenamiento se contó con 7 aparatos de electro-estimulación programables, marca Cefar Myo4, con una forma de impulso rectangular bifásica asimétrica, una intensidad de los impulsos entre 0 y $120 \mathrm{~mA}$, en cada uno de sus cuatro canales y una anchura del impulso o duración calculada en microsegundos $(\mu \mathrm{s})$. La frecuencia del impulso es programable en un rango comprendido entre 0 y $120 \mathrm{~Hz}$. 


\section{Procedimiento de medición}

Los test de evaluación se realizaron en tres momentos: el pre-test se realizó en una sesión previa a la primera sesión de entrenamiento; el post-test 1 inmediatamente después de la última sesión de entrenamiento y el re-test, tras dos semanas de inactividad para comprobar la pérdida en cada uno de los grupos de entrenamiento. Para la evaluación del rango de movimiento activo y pasivo se utilizó los test de elevación activa y pasiva de extremidad inferior en extensión (ASLRT y PSLRT, respectivamente). Los ángulos se midieron a través de la digitalización de los puntos anatómicos con el software ATD 2.0 para Windows (Analysis of Sport Techniques, program Granada University, Spain), utilizando las fotografías tomadas durante los test de evaluación. El ángulo $(\alpha)$ se obtuvo digitalizando 3 puntos: el tobillo (maléolo), la cadera (trocánter mayor) y el tobillo de la otra extremidad inferior (maléolo). Cada fotografía fue digitalizada 3 veces en ASLRT y PSLRT, considerando para el análisis estadístico el valor promedio en cada caso. Durante los test, los participantes permanecieron tendidos sobre un banco, indicándoles que mantuvieran la cabeza alineada con la espalda, y presionando la zona lumbar contra el banco durante todo el ensayo. Manteniendo las rodillas completamente extendidas, cada participante procedió a levantar lentamente la extremidad inferior hacia arriba, flexionando la cadera evitando rotaciones internas y externas de la extremidad o desviaciones del plano sagital. Cuando se alcanzó la ROM máxima, activa o pasiva (según proceda), indicada oralmente por el participante, se mantuvo la posición y se tomó una fotografía con la cámara digital perpendicular al participante a una distancia de 4 metros, con el centro de enfoque en la articulación de la cadera.

Las fotografías se obtuvieron con una resolución de 1024x768 pixeles.

\section{Protocolo de entrenamiento}

Los participantes de los dos grupos experimentales realizaron un entrenamiento de flexibilidad de 3 sesiones por semana, durante 4 semanas. Antes de cada sesión de entrenamiento se realizó un calentamiento estandarizado durante 20 minutos, manteniéndose el mismo para todas las sesiones de entrenamiento y para ambos grupos. Las pruebas de evaluación en el pre-test, post-test y re-test y las sesiones de entrenamiento tuvieron lugar a la misma hora y en el mismo lugar, con una temperatura ambiental de $23^{\circ}$. El ejercicio de estiramiento utilizado fue la elevación de la extremidad inferior extendida (SLR, Straight-Leg-Raise). La sesión se utilizó como primer contacto y familiarización con la prueba de evaluación y la técnica empleada.

Grupo experimental 1 (CRAC+EE) (11 participantes): a este grupo se le aplicó la técnica CRAC con electroestimulación, consistente en una serie de 10 repeticiones del siguiente ciclo: elongación activa (AE) del grupo de músculos isquiotibiales hasta alcanzar el máximo ROM; contracción concéntrica máxima (CC) del músculo a estirar (isquiotibiales). Para provocar dicha contracción se aplicó una corriente eléctrica bipolar (100 mA), a través de dos electrodos de 
superficie ubicados en los extremos proximal y distal de la musculatura isquiotibial. Los parámetros de corriente utilizados fueron los siguientes: Frecuencia del impulso eléctrico $(\mathrm{FI})$ de $80 \mathrm{~Hz}$, con un tiempo de contracción (TC) de $6 \mathrm{~s}$, mientras que un auxiliar ayudaba a mantener la posición de la pierna; relajación de la contracción durante $2 \mathrm{~s}$ y contracción de los músculos opositores (cuádriceps y psoas) a la vez que se espira, ampliando el estiramiento de los isquiotibiales de forma activa hasta alcanzar un nuevo rango de movimiento, manteniendo la posición durante 10 segundos; relajación de la musculatura en la posición inicial durante $2 \mathrm{~s}$. En el estiramiento, no existe tracción por parte del auxiliar. En resumen, el ciclo fue: $6 \mathrm{~s}$ de contracción más $10 \mathrm{~s}$ de estiramiento seguidos de $2 \mathrm{~s}$ de reposo. Por lo tanto, el entrenamiento fue: 1x10x (CC $6 \mathrm{~s}+$ EA $10 \mathrm{~s}$ ) con $2 \mathrm{~s}$ de reposo entre repeticiones, resultando un tiempo total de estiramiento de $100 \mathrm{~s}$ por sesión y un tiempo de trabajo total de $3 \mathrm{~min}$.

Grupo experimental 2 (CRAC) (11 participantes): la técnica aplicada fue la misma, pero sin utilizar electroestimulación durante la contracción concéntrica (CC) del músculo a estirar (isquiotibiales), durante $6 \mathrm{~s}$.

\section{Análisis estadístico}

Previamente a su análisis, se comprobaron los supuestos de normalidad y homocedasticidad mediante los estadísticos de Shapiro Wilk y Levene, respectivamente.

Se analizaron las diferencias observadas en cada una de las variables dependientes AROM y PROM mediante un ANOVA factorial mixto o 'split-plot' (Tratamiento $x$ Medida), con tres niveles en el factor inter-sujetos (control, CRAC+EE y CRAC) y tres niveles en el factor intra-sujetos (pre-, post- y re-test).

Se comprobó la igualdad de las matrices de varianzas y covarianzas de los niveles del factor intra-sujeto en cada uno de los niveles de los factores intersujeto mediante la prueba de esfericidad de Mauchly, utilizándose el estadístico F univariado, aplicando la estimación Greenhouse-Geisser del índice corrector Épsilon en caso de incumplimiento del supuesto de esfericidad.

En las comparaciones múltiples referidas a los efectos intra-sujetos se ajustaron los niveles críticos y los intervalos de confianza mediante la corrección de Bonferroni.

Para evaluar las diferencias entre las ganancias producidas entre las medidas Pre- y Pos-test, y Post- y Re-test, en cada uno de los Tratamiento aplicados, se utilizó un ANOVA de una vía. Las comparaciones Post Hoc se realizaron utilizando el estadístico de Bonferroni. El nivel de significación usado en todos los test fue $p<.05$. Los datos fueron analizados utilizando el software IBM SPSS Statistics V.22.0 (SPSS Inc., Chicago, IL, USA).

\section{RESULTADOS}

Todas las distribuciones de los valores correspondientes a las 
subpoblaciones resultantes de combinar los distintos niveles del factor Medida y Tratamiento mostraron un comportamiento normal, $\mathrm{p}<.05$.

En la Tabla 1 se muestran los valores medios y desviaciones estándar de las distintas medidas realizadas de AROM y PROM.

Tabla 1. Valores medios (y desviación estándar) de los rangos de movimiento de flexión de cadera, activo y pasivo, medidos en grados, según los distintos grupos experimentales y niveles del factor Medida (pre-, post- y retest).

\begin{tabular}{|c|c|c|c|c|c|}
\hline & & Control & $\mathrm{CRAC}+\mathrm{EE}$ & CRAC & Total \\
\hline \multirow{6}{*}{$\begin{array}{l}\text { ROM } \\
\text { Activo }\end{array}$} & $\mathrm{N}$ & 10 & 12 & 12 & 34 \\
\hline & Pre- & $94,70(12,06)$ & $91,00(10,26)$ & $90,83(6,58)$ & $92,03(9,60)$ \\
\hline & Post- & $95,60(11,82)$ & $98,83(8,26)^{\star \star \star}$ & $96,17(5,44)^{* * \star}$ & $96,94(8,53)^{* * *}$ \\
\hline & Re-test & $95,40(11,91)$ & $94,42(8,97)^{\star \star}+t \dagger$ & $93,58(5,38)^{*} \dagger$ & $94,41(8,70)^{* * *}+t \dagger$ \\
\hline & $\begin{array}{l}\text { Ganancia } \\
\text { (Post-Pretest) }\end{array}$ &, $90(3,51)$ & 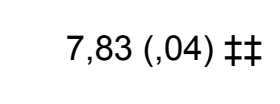 & $5,33(4,29) \ddagger$ & $4,91(4,79)$ \\
\hline & $\begin{array}{l}\text { Ganancia } \\
\text { (Post-Retest) }\end{array}$ &,$- 2(2,78)$ & 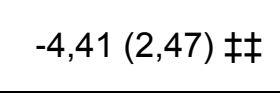 & $-2,58(3,26)$ & $2,53(3,26)$ \\
\hline \multirow{6}{*}{$\begin{array}{l}\text { ROM } \\
\text { Pasivo }\end{array}$} & $\mathrm{N}$ & 10 & 12 & 12 & 34 \\
\hline & Pre- & $118,40(24,57)$ & $120,17(20,40)$ & $116,50(9,95)$ & $118,35(18,41)$ \\
\hline & Post- & $124,00(20,89)$ & $136,33(12,52)^{\star \star *}$ & $127,92(12,01)^{* *}$ & $129,74(15,69)^{* * * t}$ \\
\hline & Re-test & $119,50(23,26)$ & $132,17(13,32)^{\star \star *}$ & $122,25(7,75)^{\star} \dagger$ & $124,94(16,04)^{* * \star}+\dagger$ \\
\hline & $\begin{array}{l}\text { Ganancia } \\
\text { (Post-Pretest) }\end{array}$ & $5,60(10,20)$ & $16,17(10,51)$ & $11,42(9,24)$ & $11,38(10,59)$ \\
\hline & $\begin{array}{l}\text { Ganancia } \\
\text { (Post-Retest) }\end{array}$ & $4,50(10,30)$ & $4,17(1,59)$ & $5,67(6,76)$ & $4,79(6,74)$ \\
\hline
\end{tabular}

${ }^{* * *}=p<, 001 ;{ }^{* *}=p<, 01 ;{ }^{*}=p<, 05 ;$ diferencias estadísticamente significativas con Pre-test ††† $=\mathrm{p}<, 001 ; \dagger \dagger=\mathrm{p}<, 01 ; \dagger=\mathrm{p}<, 05$; diferencias estadísticamente significativas con Post-test $\ddagger \ddagger=p<, 01 ; \ddagger=p<, 05$; diferencias estadísticamente significativas con Grupo Control $\mathrm{CRAC}+\mathrm{EE}=$ Contract-Relax Antagonist Contract with Electrostimulation CRAC $=$ Contract-Relax Antagonist Contract

El análisis factorial mixto mostró tendencias similares para los rangos de movimiento activo y pasivo.

\section{Rango de Movimiento Activo (AROM)}

En relación a AROM, el ANOVA de medidas repetidas mostró un efecto principal estadísticamente significativo del factor Medida, $F(2,62)=32,255, p=$ ,000, $\eta_{p}^{2}=, 510$, así como de la interacción Tratamiento*Medida, $F(4,62)=5,872$, $p=, 000, \eta_{p}^{2}=, 275$.

Los valores medios de AROM (véase Tabla 1) fueron estadísticamente superiores en las medidas Post- y Retest respecto a la medida Pre- $(p<, 001$, $95 \%$ IC $[2,951,6,427]$ y $p=, 001,95 \%$ IC $[, 882,3,696]$, respectivamente), y la medida Post- superior a la medida Retest $(p=, 000,95 \% \mathrm{IC}[1,154,3,646])$.

Respecto a la interacción Tratamiento*Medida, el análisis estadístico 
destacó como el rango de movimiento activo fue significativamente diferente entre las distintas medidas según se aplicase CRAC+EE y CRAC.

Aplicando la técnica $\mathrm{CRAC+EE}$, los valores promedio de la medida Prefueron significativamente inferiores a los obtenidos en las medias Post- y Retest $(p<, 001,95 \% \mathrm{IC}[4,919,10,748]$ y $p=, 003,95 \% \mathrm{IC}[1,057,5,776]$, respectivamente). Y el valor medio de la medida Post- significativamente superior al registrado para la medida Retest $(p<, 001,95 \%$ IC $[2,328,6,506])$.

Esta misma dinámica se observó cuando se aplicó la técnica CRAC. EI valor promedio de la medida Post- fue significativamente superior a los obtenidos en las medidas Pre- y Retest, $(p<, 001,95 \% \mathrm{IC}[2,419,8,248]$ y $p=, 011,95 \% \mathrm{IC}$ [,494, 4,672], en el mismo orden) y éste último igualmente superior al obtenido en la medida Pre- $(p=, 011,95 \%$ IC [,494, 4,672]) (véase Figura 1).

Atendiendo al factor intra-grupo 'Tratamiento', no se observaron diferencias estadísticamente significativas entre los valores promedio totales del rango de movimiento activo, indistintamente de los pares de niveles contrastados, $F(1,31)=, 109, p=, 897, \eta_{p}^{2}=, 007$.
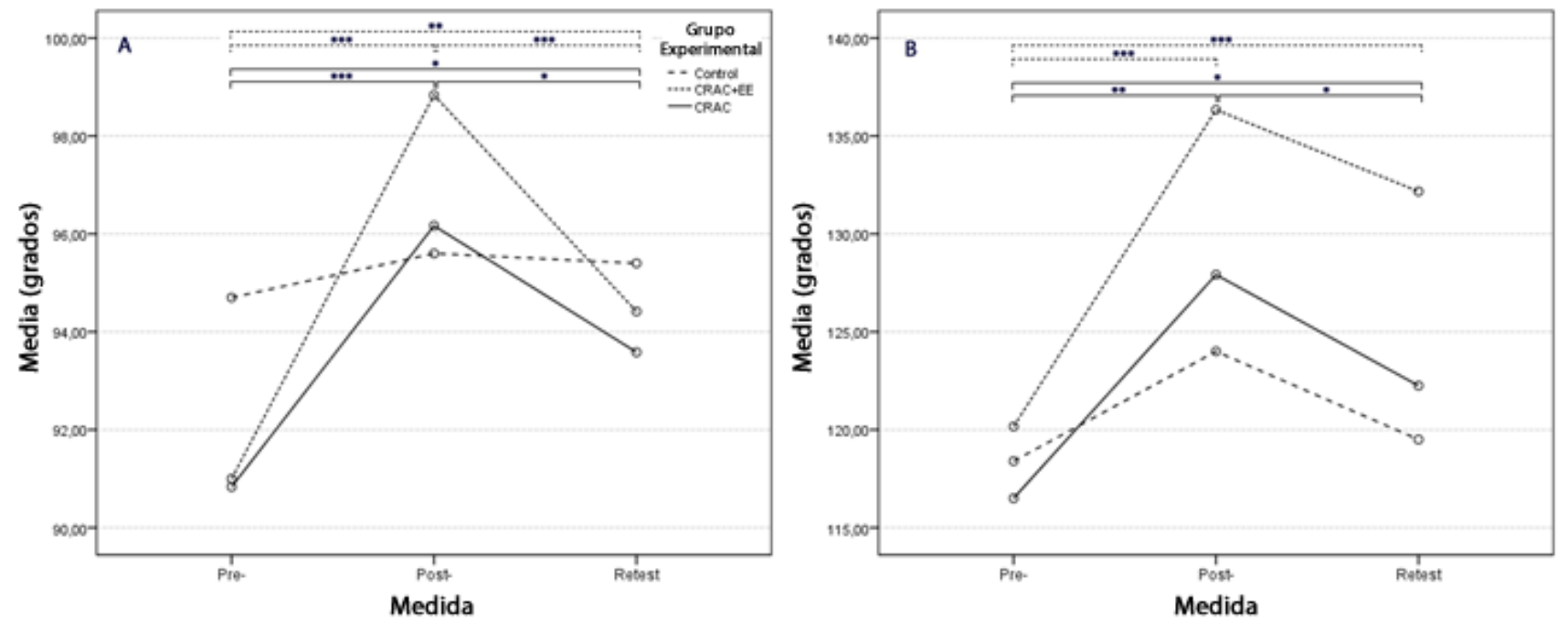

Figura 1. Valores promedio de AROM (A) y PROM (B) según las distintas medidas realizadas, para cada uno de los grupos experimentales (Control, Contract-Relax AntogonistContract con electroestimulación - CRAC+EE y Contract-Relax Antogonist-Contract - CRAC).

\section{Rango de movimiento Pasivo (PROM)}

El ANOVA Split-Plot indicó un efecto principal significativo del factor Medida, $F(1,622,50,287)=30,818, p=, 000, \eta_{p}^{2}=, 499$. En concreto, los valores promedio de la medida Pre-test fueron significativamente menores que los obtenidos para las medidas Post- y Retest $(p=, 000,95 \% \mathrm{IC}[6,711,15,411]$ y $p$ $=, 000,95 \% \mathrm{IC}[3,064,9,503]$, respectivamente). La medida promedio Post- fue significativamente superior a la registrada en la medida Retest $(p=, 001,95 \% \mathrm{IC}$ $[1,763,7,793])$ (véase Tabla 1).

Asimismo, se observó un efecto significativo de la interacción 
Tratamiento*Medida, $\left.F(3,244,50,287)=3,177, p=, 029, \eta_{p}^{2}=, 170\right)$, centrándose las diferencias detectadas en los tratamientos CRAC+EE y CRAC (véase Figura 2).

En el caso de CRAC+EE, las diferencias entre los distintos pares mostraron valores medios menores de la medida Pre- con respecto a las tomas Post- y Retest $(p=, 000,95 \% \mathrm{IC}[8,871,23,462]$ y $p=, 000,95 \% \mathrm{IC}[6,600,17,400]$, en el mismo orden). La aplicación de CRAC generó valores promedio superiores de ROM en las medidas Post- y Re-test respecto a la medida Pre-test $(p=, 001$, $95 \%$ IC $[4,121,18,712]$ y $p=, 034,95 \%$ IC $[, 350,11,150]$, respectivamente), así como mayores valores promedio de la medida Post- respecto a la medida Retest $(p=, 024,95 \% \mathrm{IC}[, 610,10,723])$.

No se observaron diferencias estadísticamente significativas entre los valores totales promedio de las distintas tomas asociadas al factor intra-grupo Tratamiento, $F(2,31)=1,014, p=, 374, \eta_{p}^{2}=, 061$.

\section{Ganancia producida entre las tomas Pre-Postest y Post-Retest}

Considerando el Rango de Movimiento Activo, el análisis de varianza de una vía detectó diferencias estadísticamente significativas en la ganancia producida entre las tomas Pre- y Pos-test, $F(2,31)=8,343, p=, 001$, y las tomas Post- y Re-test, $F(2,31)=5,935, p=, 007$ (véase Figura 3).

En el primero de los casos, las pruebas Post Hoc indicaron que la diferencia obtenida entre las tomas Pre- y Pos-test en el grupo Control resultó significativamente inferior a la obtenida en los tratamientos CRAC+EE, $p=, 001$, $95 \%$ IC $[2,6107,11,2559]$ y CRAC, $p=, 043,95 \%$ IC $[, 1107,8,7559]$. Respecto a la ganancia producida entre las tomas Post-Retest, la única diferencia significativa se observó entre los tratamientos Control y CRAC+EE, $p=, 005$, $95 \%$ IC [1,1182, 7,3152], siendo los valores absolutos de las diferencias promedio obtenidas mayores en el grupo CRAC+EE (véase Tabla 1). 

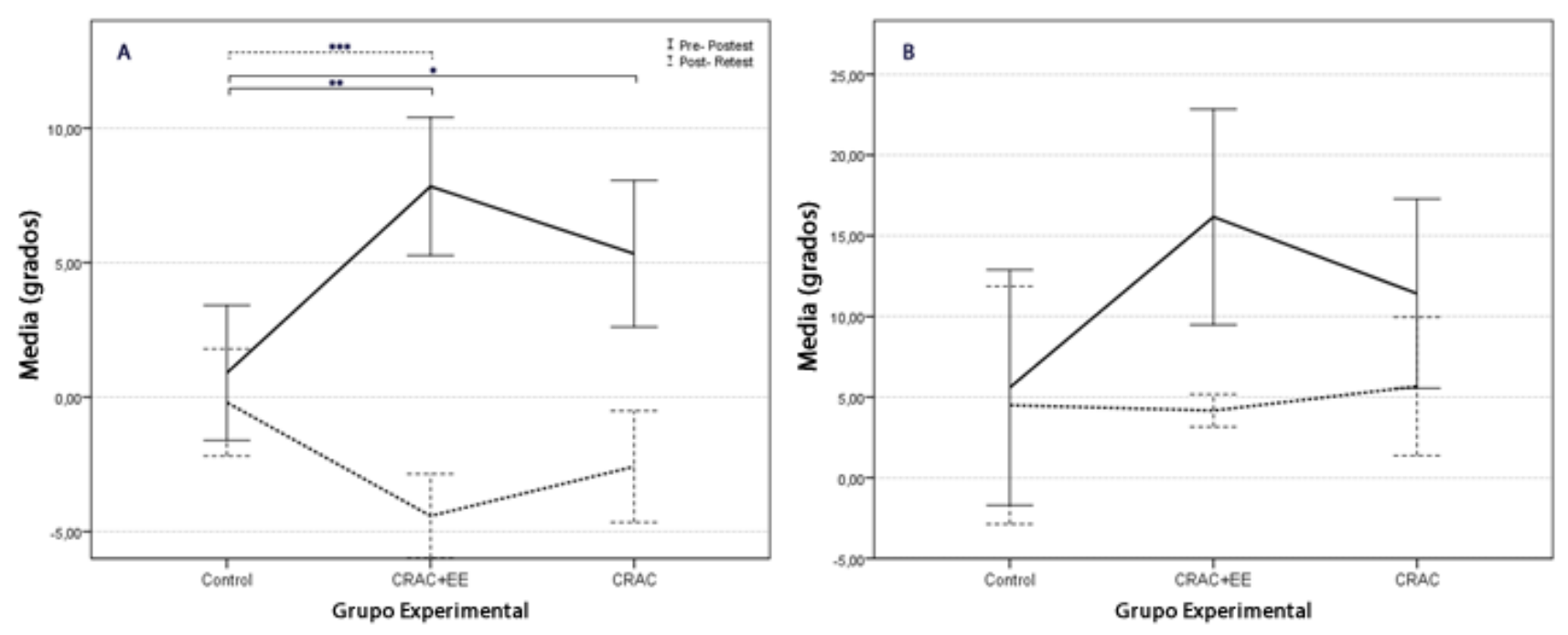

Figura 2. Mejora producida, expresada en grados, entre las tomas Pre-Post test y Post-Retest en AROM (A) y PROM (B) según los distintos tratamientos aplicados.

$\mathrm{CRAC}+\mathrm{EE}=$ Contract-Relax Antagonist Contract with Electrostimulation; $\mathrm{CRAC}=$ Contract Relax Antagonist Contract

${ }^{* * *}=\mathrm{p}<.001 ;{ }^{* *}=\mathrm{p}<.01 ;{ }^{*}=\mathrm{p}<.05 ;$ Barras de error $95 \%$ IC

No se observaron diferencias estadísticamente significativas entre las diferencias obtenidas en PROM, entre los pares de medidas Pre-Postest, $F(2$, $31)=3,054, p=, 062$ y Post-Retest, $F(2,31)=, 154, p=, 858$, según los tres niveles del factor Tratamiento (Figura 4).

En ningún caso, la ganancia producida entre las tomas Pre-Postest y Post-Retest fue significativamente diferente entre los tratamientos CRAC+EE y CRAC.

\section{DISCUSION}

Los mayores hallazgos obtenidos en este estudio fueron una mejora significativa del ROM pasivo y activo en los dos grupos experimentales con y sin inducción de contracción mediante electroestimulación, mientras el grupo control mantuvo prácticamente sus condiciones iniciales.

Las mejoras de los grupos CRAC+EE y CRAC fueron $16,17^{\circ}$ y $11,42^{\circ}$ respectivamente para PROM y $7,83^{\circ}$ y $5,33^{\circ}$ para AROM tras una intervención de 3 sesiones semanales durante 4 semanas (Tabla 1). Estos resultados confirman los encontrados en otros estudios (Etnyre y Abraham, 1986; McAtee y Charland, 2000) en relación al entrenamiento utilizando la técnica CRAC, quienes tras un periodo de entrenamiento obtuvieron mejoras tanto en el ROM pasivo como activo.

Los trabajos de Etnyre y Abraham (1986) y Sundquist (1996) al comparar la técnica de Estiramiento Estático y distintas técnicas de FNP, CR y CRAC, concluyen que la técnica CRAC es la que genera una mayor influencia neuronal inhibitoria de la actividad motora durante el estiramiento, reduciendo la 
contractibilidad muscular de la musculatura a estirar, lo que permite una mayor obediencia muscular, y la obtención de un mejor incremento en el ROM.

El estudio realizado por Etnyre y Abraham (1986) muestra resultados medios de $26^{\circ}$ de mejora en el rango de movimiento de la cadera tras un periodo de entrenamiento de 12 semanas con la técnica CRAC. Son valores referidos a manifestaciones pasivas y aunque se encuentran por encima de los obtenidos por los dos grupos experimentales de nuestro estudio, se trata de un periodo de entrenamiento 3 veces superior. En dicho estudio, se trabajó con tiempos de contracción igual al nuestro (6 s), pero con estiramientos de 3 s durante 12 semanas, pudiendo ser ésta la causa por la que los márgenes conseguidos no son comparables con el nuestro, (12 semanas frente a 4 en nuestro estudio).

Igualmente se constata la eficacia de la electroestimulación para la mejora del ROM, como en los estudios realizados a largo plazo con utilización de técnicas diferentes y aplicación de corrientes variadas (Acosta et al., 1998; Pérez y Álamo, 2001; Basas, 2001; López-Bedoya et al., 2002; Maciel y Câmara, 2008; Espejo et al., 2012).

Destacar la efectividad de la técnica CRAC combinada con electroestimulación encontrada en el estudio de López-Bedoya et al. (2002). En dicho trabajo se compararon igualmente dos protocolos con y sin inducción de contracción muscular mediante electroestimulación utilizando la técnica CRAC, encontrándose mejores resultados para la técnica con electroestimulación, en el postest, tanto en las manifestaciones pasivas como activas, sin diferencias estadísticamente significativas entre ambos como ocurre en nuestro estudio.

Maciel y Câmara (2008) evaluaron el efecto asociado de la electroestimulación con estiramientos pasivos (EP) para lograr ganancias de flexibilidad en los músculos isquiotibiales. Los resultados mostraron que los grupos de EP y EP+EE con el programa TENS aumentaron su rango de movimiento en relación con el grupo de control, pero no hubo diferencias entre los dos grupos, como en nuestro estudio.

No obstante, es importante resaltar que aunque el TENS obtuvo mejoras en los dos grupos experimentales como en nuestro estudio, uno de los aspectos más importante de la efectividad de los protocolos empleados al trabajar la extensibilidad muscular con electroestimulación, es la elección de corrientes idóneas, es decir, aquellas corrientes, como las manejadas en nuestro estudio que, a diferencia del TENS, poseen frecuencias capaces de estimular de manera real el músculo esquelético (Plaja 1999; Linares, Escalante y LaTouche, 2004).

Por otro lado, se corrobora también, como en otras investigaciones, que las mejoras en el PROM son siempre superiores al AROM (Roberts y Wilson, 1999; López Bedoya et al., 2013).

En este sentido, consideramos importante resaltar que las mejoras del AROM en nuestro estudio pueden deberse a la técnica de CRAC utilizada, ya 
que son varios los trabajos que demuestran que las mejoras de las manifestaciones activas, van asociados a técnicas de FNP tipo CRAC, técnica utilizada en este estudio (Etnyre y Abraham, 1986; Sundquist, 1996; McAtee y Charland, 2000; Rowlands, Marginson y Lee, 2003; Sharman, Cresswell y Riek, 2006).

Muchos de estos estudios precedentes explican estas ganancias por el mecanismo de inervación recíproca. En la técnica CRAC, el músculo que se contrae "músculo opositor" al estiramiento, provoca ese reflejo e inhibe la musculatura a estirar (ME). Esta inhibición de la ME, junto con la contracción acortamiento del músculo opositor, permite que las fibras musculares de la ME se alarguen aún más, debido a que se produce una influencia inhibidora más grande en dicha musculatura (Etnyre y Abraham, 1986; Sharman et al., 2006). Las interneuronas que inervan las alfa-motoneuronas, que sinapsan en la ME, hacen que la actividad neural en dicha musculara disminuya, lo que conduce a un mayor estiramiento en la misma (Rowlands et al., 2003).

En cuanto a los resultados del re-test, en relación al PROM, en ningún caso, la ganancia producida entre las tomas Post-Retest fue significativamente diferente entre los tratamientos CRAC+EE y CRAC. Los datos mostraron, que los sujetos de ambos grupos experimentales que utilizaron la técnica de PNFCRAC con y sin electroestimulación, perdieron poca de la mejora obtenida en el rango de movimiento de la articulación de la cadera en comparación con el pretest; después de que se suspendiera el tratamiento durante dos semanas. Es decir, en ninguno de los grupos experimentales existió una pérdida completa, donde los valores mantenidos entre pre- y retest fueron de 5,76ํㅜ para el grupo de CRAC y de $12^{\circ}$ para CRAC+EE (véase Figura 2 ).

Los datos registrados confirman los resultados obtenidos en el estudio de Zamora y Salazar (2001); quienes afirmaron respectivamente que la técnica PNF permitía mantener por mayor período de tiempo la mejora obtenida en el rango de movimiento articular de la cadera, con respecto a la utilización de técnicas de estiramiento estáticas.

No obstante, en la revisión documental se comprueba que existen resultados contrapuestos en relación a la duración de los efectos que tiene el estiramiento utilizando técnicas de PNF sobre el ROM. Así, McCarthy, Olsen y Smeby (1997), pusieron en evidencia que las ganancias en el ROM duran aproximadamente siete días después de una semana de estiramiento dos veces al día; mientras que otros estudios indican que los incrementos del ROM decrecen con relativa rapidez una vez que cesa la intervención (Wallin, Ekblom, Grahn y Nordenborg, 1985; Spernoga, Uhl, Arnold y Gansneder, 2001; Funk, Swank, Mikla, Fagen y Farr, 2003), siendo aconsejable trabajar el estiramiento con PNF, al menos una o dos veces por semana, con el objetivo de estabilizar el ROM a largo plazo.

La duración de estos efectos puede variar debido al tiempo de estiramiento y la duración de la contracción durante el estiramiento PNF (Feland y Marín, 2004; Rowlands et al., 2003). Se ha demostrado que este tipo de 
contracción produce mejores efectos cuando se mantiene entre 3 y $10 \mathrm{~s}$, considerándose preferible un tiempo de 6 s (Feland y Marín, 2004).

En las condiciones de nuestro estudio, con una contracción de $6 \mathrm{~s}$, se constata que en ninguno de los grupos experimentales existió una pérdida completa, tras dos semanas sin aplicar las técnicas de estiramientos (véase Figuras 1 y 2). Estos resultados contradicen lo indicado por McCarthy et al., (1997), en relación a la permanencia de las ganancias del ROM, indicando que éstas se pierden una vez transcurridos siete días.

Sin embargo, es importante destacar que no es igual para el AROM donde también se encontraron ligeras pérdidas entre el post-test $\left(96,17^{\circ}\right)$ y retest $\left(93,5^{\circ}\right)$ de $2,58^{\circ}$, y entre pretest $\left(90,83^{\circ}\right)$ y retest $(93,58)$ de $2,75^{\circ}$ en CRAC (véase figura 1), sensiblemente menores al grupo tratado con la técnica CRAC+EE, con $\left(93,83^{\circ}\right)$ post-test y $\left(94,42^{\circ}\right)$ retest de $4,41^{\circ}$. Destacar que en este grupo, entre el pretest $\left(91^{\circ}\right)$ y retest $\left(94,42^{\circ}\right)$, se mantiene una ligerísima ganancia de $3,42^{\circ}$.

La reflexión a la que nos lleva estos resultados del AROM, es que la aplicación de la técnica de entrenamiento de Flexibilidad CRAC+EE, si bien produce un incremento temporal del rango de movimiento activo, transcurridas dos semanas del cese, produce un efecto adverso sobre el mismo, que conlleva una reducción de aquel inferior al que se produciría si los sujetos no hubiesen sido sometidos a tratamiento alguno (grupo control). Dicho efecto adverso no se registra en el caso de los sujetos sometidos a CRAC en los que, si bien la ganancia producida es similar al del grupo $C R A C+E E$, la reducción del rango de movimiento que conlleva permanece análoga al del grupo control.

\section{CONCLUSIONES}

Este estudio muestra como un protocolo de entrenamiento de la flexibilidad en deportistas jóvenes mediante técnicas de estiramientos FNP CRAC con y sin contracción inducida mediante electroestimulación (tres sesiones por semana durante cuatro semanas, con un tiempo de estiramiento total 100 segundos por sesión) sobre la musculatura isquiotibial, fue efectivo en la mejora del Rango de Movimiento Activo y Pasivo. Aunque no existen diferencias significativas entre ambas técnicas, los resultados indican que hay una ligera tendencia hacia una mayor ganancia del ROM en CRAC+EE frente a CRAC. Igualmente, ambas técnicas permiten mantener la mejora del AROM y PROM con un ligero descenso tras dos semanas de inactividad.

En definitiva, el entrenamiento de la flexibilidad mediante técnicas de estiramientos FNP CRAC con y sin contracción muscular inducida mediante electroestimulación, logra efectos beneficiosos en la mejora de la extensibilidad isquiotibial, siendo necesario continuar su investigación con muestras de tamaños superiores, diferentes franjas de edades y características variadas (deportistas de élite, de iniciación, etc.) que representen al resto de la población y con diseños metodológicos que incluyan periodos inmediatos y de seguimiento a medio y largo plazo. 
Es necesario destacar la prudencia en generalizar los resultados de este estudio a otros grupos de sujetos con características diferentes de edad, género, nivel de actividad física o nivel inicial de ROM; también a extrapolar los mismos a otros grupos musculares y puntos articulares diferentes, o en condiciones ambientales distintas, lo cual supone la continuación de nuevos retos en investigaciones futuras a realizar

\section{Aplicaciones prácticas}

Como consecuencia de las conclusiones obtenidas, se podría considerar que el entrenamiento de la flexibilidad en deportistas mediante técnicas de FNP que incluyen un estiramiento activo, tipo CRAC con y sin electroestimulación son indicadas tanto para la mejora del Rango de movimiento activo como pasivo.

Por otro lado, teniendo en cuenta la eficacia de estas técnicas, su uso en los deportes artísticos-expresivos como la gimnasia rítmica y artística, natación sincronizada, patinaje artístico etc., podría proporcionar efectos beneficiosos ya que tener grandes rangos de movimiento en posturas inusuales es un factor clave en el rendimiento de los mismos (Sands, 2002).

\section{REFERENCIAS BIBLIOGRÁFICAS}

Acosta, J.; López Bedoya, J. \& Vernetta, M. (1998). Mejora del Spagat frontal aplicando métodos de Facilitación Neuromuscular Propioceptiva y técnicas de Electroestimulación y Biofeedback. En M. Vernetta, J. López-Bedoya \& P. Panadero (eds.) Novedades en Actividades Gimnásticas (pp. 127-130). Granada: Editores.

Adler, S. S.; Berkers, D. \& Buck, M. (2002). La Facilitación Neuromuscular Propioceptiva en la práctica. Guía ilustrada. Buenos Aires: Editorial Panamericana.

Alter, M. J. (2004). Science of Flexibility ( $3^{\underline{a}}$ Ed.). Champaign: Human Kinetics.

Ayala, F., Sainz de Baranda, P. \& Cejudo, A. (2012). Flexibility training: Stretching techniques. Revista Andaluza de Medicina del Deporte, 5, 105112. https://doi.org/10.1016/S1888-7546(12)70016-3

Barnett, A. (2006). Using recovery modalities between training sessions in elite athletes: does it help? Sports Medicine, 36(9), 781-796. https://doi.org/10.2165/00007256200636090-00005

Basas, G. A. (2001). Metodología de la electroestimulación en el deporte. Fisioterapia, 23, 36-47. https://doi.org/10.1016/S0211-5638(01)72971-9

De Hoyo, M. \& y Sañudo, B. (2006). La electroestimulación como mejora de la flexibilidad. http://www.efdeportes.com/ Revista Digital - Buenos Aires, 11 (101).

Etnyre, B.R. \& Abraham, L.D. (1986). H-reflex changes during static stretching and two variations of proprioceptive neuromuscular facilitation techniques. Electroencephalography and Clinical Neurophysiology, 63, 174-179. https://doi.org/10.1016/0013-4694(86)90010-6

Espejo, L., Maya, J., Cardero, M.A., \& Albornoz, M. (2012). Aumento de la extensibilidad isquiotibial tras aplicar elongación muscular eléctrica. Fisioterapia. 34(3), 112-117. https://doi.org/10.1016/..ft.2012.01.001

Feland, J.B., \& Marin, H.N. (2004). Effect of submaximal contraction intensity in contractrelax proprioceptive neuromuscular facilitation stretching. British Journal of Sports 


\section{Medicine, 38(4):e18. https://doi.org/10.1136/bjsm.2003.010967}

Funk, D.C., Swank, A.M., Mikla, B.M., Fagen, T.A., \& Farr, B.K. (2003). Impact of Prior Exercise on Hamstring Flexibility: A Comparison of Proprioceptive Neuromuscular Facilitation and Static Stretching. National Strength \& Conditioning Association Journal. 17(3), 489-492. https://doi.org/10.1519/00124278-200308000-00010

García-Manso, J.M., López-Bedoya, J., Rodríguez-Matoso, D., Ariza, L., RodríguezRuiz, D. \& Vernetta, M. (2015). Static-stretching vs. contract-relax - proprioceptive neuromuscular facilitation stretching: study the effect on muscle response using tensiomyography. European Journal of Human Movement, 34, 96-108.

Kenric, L. (2003). A Comparison of Different Methods for Improving Hamstring Flexibility. Tesis Doctoral. Florida Atlantic University.

Linares, M.; Escalante, K., \& LaTouche, R. (2004). Revisión bibliográfica de las corrientes y parámetros más efectivos en la electroestimulación del cuádriceps. Fisioterapia, 26(4), 235-244. https://doi.org/10.1016/S0211-5638(04)73108-9

López-Bedoya, J.; Goméz-Landero, A.; Jiménez, J. \& Vernetta, M. (2002). Estudio comparativo entre técnicas de Facilitación Neuromuscular Propioceptiva mediante electroestimulación (FNP-EEM) y secuencia estiramiento facilitado Contracción, Relajación, Contracción Antagonista (CRCA) en la musculatura isquiotibial. En Enseñanza y entrenamiento de la Gimnasia y la Acrobacia. Actas del I Simposium Internacional de Actividades Gimnásticas y Acrobáticas (pp 160-165). Cáceres: Universidad de Extremadura.

López-Bedoya, J., Vernetta, M., Robles, A \& Ariza, L. (2013). Effect of three types of flexibility training on active and passive hip range of motion. The Journal of Sports Medicine and Physical Fitness, 53(3), 304-11.

Maciel, A.C.C. \& Câmara, S.M.A. (2008). Influência da estimulação elétrica nervosa transcutânea (TENS) associada ao19.alongamento muscular no ganho de flexibilidade. Revista Brasileira de Fisioterapia, 5(12), 373-378. https://doi.org/10.1590/S1413-35552008000500006

Maffiuletti, N.; Dugnani, S.; Folz, M.; Di Pierno, E., y Mauro, F. (2002). Effects of combined electrostimulation and plyometric training of vertical jump height. Medicine and Science in Sports and Exercise, 34(10), 1638-1644. https://doi.org/10.1097/00005768-200210000-00016

Martin, V.G., Millet, G.Y., Lattier, G. \& Perrod, L. (2004). Effects of recovery modes after knee extensor muscles eccentric contractions. Medicine and Science in Sports and Exercise, $36(11)$, https://doi.org/10.1249/01.MSS.0000145526.43208.08

McAtee, R.E. \& Charland, J. (2000). Estiramientos Facilitados. Los estiramientos de FNP con y sin asistencia. Barcelona: Paidotribo.

McCarthy P.W., Olsen J.P. \& Smeby I.H. (1997). Effects of contract-relax stretching procedures on active range of motion of the cervical spine in the transverse plane. Clinical Biomechanics, 12 (2), 136-138. https://doi.org/10.1016/S0268$\underline{0033(96) 00060-5}$

Naffiuletti, N.A., Zory, R., Miotti, D., Pellegrino, M.A, Jubeau, M., Bottinelli, R. (2006). Neuromusuclar adaptations to electrostimulation resistance training. American Journal of Physical Medicine \& Rehabilitation, 85(2), 167-75. https://doi.org/10.1097/01.phm.0000197570.03343.18

Norris, C.M. (2007). La Guía completa de los estiramientos. 2ª ed. Barcelona: Paidotribo. Page, P. (2012).Current concepts in muscle stretching for exercise and rehabilitation. The International Journal of Sports Physical Therapy, 7(1), 109-119.

Pérez, M.J. \& Álamo, A.D. (2001). Comparative study between muscular stretching by active tension and electrostimulation. Fisioterapia, 23, 10-14. 


\title{
https://doi.org/10.1016/S0211-5638(01)72924-0
}

Plaja, J. (1999) Guía práctica de electroterapia. Barcelona: Carin Electromedicarin, SA.

Pombo, M., Rodríguez, J., Bruñe, X. \& Requena, B. (2004) La electroestimulación entrenamiento y periodización. Barcelona: Paidotribo.

Roberts, J.M. \& Wilson, K. (1999). Effect of stretching duration on active and passive range of motion in the lower extremity. British Journal of Sports Medicine, 33(4):259-63. https://doi.org/10.1136/bjsm.33.4.259

Rowlands, A.V., Marginson, V. \& Lee, J. (2003). Chronic flexibility gains: effect of isometric contraction duration during proprioceptive neuromuscular facilitation stretching techniques. Research Quarterly for Exercise and Sport, 74(1), 47-52. https://doi.org/10.1080/02701367.2003.10609063

Sands, WA. (2002). Physiology. In W.A. Sands, D.J. Caine \& J. Borms, (eds.) Scientific Aspects of Women's Gymnastics (pp.128-161). Switzerland: Karger Publisher. https://doi.org/10.1159/000067490

Sharman, M.J., Cresswell, A.G. \& Riek, S. (2006). Proprioceptive Neuromuscular Facilitation Stretching Mechanisms and Clinical Implications. Sports Medicine, 36(11), 929-939. https://doi.org/10.2165/00007256-200636110-00002

Spernoga, S.G., Uhl, T.L., Arnold, B.L. \& Gansneder, B.M. Duration of Maintained Hamstring Flexibility After a One-Time, Modified Hold-Relax Stretching. Protocol. Journal of Athletic Training, 36(1), 44-48.

Sundquist, R.D. (1996). The comparative effectiveness of static stretching and propioceptive neuromuscular facilitation stretching techniques in increasing hip flexion range of motion. Microform publications, for Sport \& Human Performance, Universidad de Oregon.

Surburg, P. R. \& Schrader, J. W. (1997). Proprioceptive Neuromuscular Facilitation Thechniques in Sports Medicine: A Reassessment. Journal of Athletic Training, 31(1), 34-39.

Voss, D. E., Ionta, M. K. \& Meyers, B. J. (2004). Facilitación Neuromuscular Propioceptiva. Patrones y técnicas. Buenos Aires: Editorial Panamericana.

Wallin, D., Ekblom, B., Grahn, R., \& Nordenborg, T. (1985). Improvement of muscle flexibility: A comparison between two techniques. The American Journal of Sports Medicine, 13(4), 263-268. https://doi.org/10.1177/036354658501300409

Zamora, J.D. \& Salazar W. (2001). Efecto del método de estiramiento PNF y estático en la mejora de la flexibilidad de la articulación de la cadera en practicantes de kung Fu. Revista de Ciencias del Ejercicio y la Salud, 1(2), 43-50. https://doi.org/10.15517/pensarmov.v1i2.430

\footnotetext{
Número de citas totales / Total references: 37 (100\%)

Número de citas propias de la revista / Journal's own references: $0(0 \%)$
}

\author{
Rev.int.med.cienc.act.fís.deporte - vol. 19 - número 74 - ISSN: 1577-0354
}

